\title{
Deformation of the notochord by pressure from the swim bladder may cause malformation of the vertebral column in cultured Atlantic cod Gadus morhua larvae: a case study
}

\author{
Sindre Grotmol*, Harald Kryvi, Geir K. Totland \\ Department of Biology, University of Bergen, Allégt. 41, 5007 Bergen, Norway
}

\begin{abstract}
This study describes a malformation that frequently occurs in Atlantic cod Gadus morhua in intensive culture systems. The malformation is characterised by a slight upward tilt of the head and an indented dorsal body contour at the transition between the head and the trunk, and is first evident to the fish farmer when the cod reach the juvenile stage. These abnormalities are associated with malformations of the neurocranium, the cranial region of the vertebral column and the cranial part of the epaxial lateral muscles. The pathogenesis involves deformation of the notochord, which can be observed in larvae about $7 \mathrm{~d}$ post-hatch (dph) and onwards. The deformation consists of an increase in dorsal curvature of the notochord in the region above the swim bladder. In the same region, the notochord has an abnormal cross-sectional outline, characterised by a groove-shaped, longitudinal impression along the ventral surface of the sheath. In most cases, the swim bladder fills the impression, and in severely affected larvae it forms a hernia-like lesion in the notochord. The deformation of the notochord seems to be conveyed to the vertebral body anlagen (chordacentra), which in teleosts are formed by mineralisation within the notochordal sheath. The vertebral bodies adopt an abnormal wedge shape, with a ventral concavity, and the neural arches are most often S-shaped. A continuous range of degrees of the malformation can be observed. All these pathomorphological characteristics are compatible with the notion that the notochord has been subjected to an upward mechanical force, probably generated by a persistent increase in pressure between the swim bladder and the notochord during the period of development of the vertebral anlagen. Our results thus indicate that the critical time window with regard to development of the malformation is from 18 to $36 \mathrm{dph}$, when the initial formation of the vertebrae takes place. Chronic overinflation of the swim bladder or pathological dilatation of the digestive tract may cause the lesions, and aetiology may be related to factors that influence the function of these organs.
\end{abstract}

KEY WORDS: Cod larvae · Notochord · Deformation · Vertebral column · Malformation · Swim bladder $\cdot$ Intensive culturing

\section{INTRODUCTION}

Commercial interest in the farming of Atlantic cod Gadus morhua has increased in the course of the past few years, and a number of large-scale, land-based facilities for intensive production of juveniles have been established in the north Atlantic region. The adaptability of cod to intensive culture conditions has been demonstrated in a number of studies (Laurence 1978, Howell 1984, Rosenlund et al. 1993, BaskervilleBridges \& Kling 2000a), but average survival rates are still low. Peak mortality rates are observed during the first feeding and weaning periods, and optimisation of diets, feeding regimes and culture conditions might still have the potential to increase survival rates (Baskerville-Bridges \& Kling 2000a,b, Shields et al. 
2003). A problem encountered in many Norwegian production facilities is the presence of a characteristic malformation of the vertebral column in juvenile cod. The malformation is characterised by a slight upward tilt of the head, associated with an abnormal dorsal contour of the cranial part of the epaxial musculature (see Fig. 1), and may first be observed by visual examination when the fish have reached a weight of about $5 \mathrm{~g}$. The incidence varies between batches, from a small fraction to most of the batch. The malformation is a cause of concern with regard to the welfare of the fish, and the abnormal body shape may reduce the marketability of the product.

The study of the normal mechanisms of development is a prerequisite for the understanding of malformations, as these may start early in ontogenesis, during organogenesis. During the embryonic and early larval stages, the notochord constitutes the main axial support through its hydroskeletal properties. The cranial portion of the notochord is located immediately ventral to the brain and forms the floor of the neurocranium, together with the parachordal cartilages. The portion of the notochord caudal to these cartilages is subsequently incorporated in the vertebral column. The notochord is composed of a core of epitheloid cells surrounded by an acellular fibrous sheath. The cellular core comprises an outer germinal layer of chordoblasts, which rest on the sheath, and an inner layer of chordocytes, each with a fluid-filled vacuole. The hydroskeletal property of the notochord is dependent on turgor generated by expansion of the vacuoles within the confined space of the sheath. The force needed for axial compression of the notochord exceeds by far that sufficient for lateral flexion, which sustains the oscillatory, lateral swimming movements of fish larvae (Koehl et al. 2000).

The vertebral column of teleosts differentiates at a late stage, after most other organs have developed. A derived characteristic of actinopterygian fishes is that ring-shaped mineralised zones, known as chordacentra, develop segmentally within the notochordal sheath, forming the initial anlagen of the vertebral bodies (Goodrich 1930, François 1966, Arratia et al. 2001, Grotmol et al. 2003). Sclerotomal osteoblasts then condense around the notochord on the surface of the chordacentra, utilising them as foundations for further growth of the vertebrae through direct ossification (Grotmol et al. 2003). The amphicoelous-shaped vertebral bodies, together with other sclerotomal structures, such as neural and haemal arches, form the complete vertebrae (Nordvik et al. 2005).

The principal objective of this paper is to elucidate the pathogenesis of the malformation in cod through a sequential study of the morphogenesis of the skeleton from the larval to the juvenile stage.

\section{MATERIALS AND METHODS}

Fish and culture conditions. Larvae and juveniles of Atlantic cod Gadus morhua were collected from 4 different production facilities in Norway for intensive culture during the spring of 2003. All the facilities kept 4 groups of wild-caught broodstock, and artificial light regimes were employed to induce spawning at different times of the year. The fish spawned naturally in the tanks, where the eggs were collected from the outlet water and thereafter kept in small, aerated, coned tanks throughout hatching. After hatching, the larvae were transferred to $30 \mathrm{~m}^{3}$ indoor start-feeding tanks. Each facility employed different fish densities, feeding protocols and temperature regimes (Table 1).

Sampling. A series of developmental stages, from the day the larvae hatched to $90 \mathrm{~d}$ post-hatch (dph), at a sampling interval of $2 \mathrm{~d}$, was analysed from each facility. Each sample consisted of 30 fish collected at random from different depths in the tank. From each sample, 20 fish were processed as whole specimens and stained with Alizarin red (see last subsection below), and the remaining individuals were processed for histology. In addition, malformed juvenile fish weighing approximately $50 \mathrm{~g}$ were examined by macroscopic dissection and radiography. The fish were anaesthetised in water containing $50 \mathrm{ppm}$ benzocain before preparative procedures commenced.

Radiography. Right lateral radiographs of fish weighing about $50 \mathrm{~g}$ were taken using a portable Xray apparatus (HI-Ray 100, Eickenmeyer Medizintechnik für Tierärzte). The film $(30 \times 40 \mathrm{~cm}$, AGFA D4 DW ETE) was exposed twice for $50 \mathrm{~mA}$ and $72 \mathrm{kV}$, and developed using a manual developer (Cofar Cemat C56D) with Kodak Professional manual fixer and developer. The images were digitalised by scanning.

Histology. The specimens were fixed by immersion in a mixture of $10 \mathrm{ml} 10 \%$ formaldehyde (fresh from paraformaldehyde), $10 \mathrm{ml} 25 \%$ glutaraldehyde, $20 \mathrm{ml}$ $0.2 \mathrm{M}$ cacodylate buffer and $60 \mathrm{ml}$ phosphate-buffered saline, and the $\mathrm{pH}$ adjusted to 7.35 . They were then

Table 1. Overview of main production parameters, and initial density of Gadus morhua larvae and survival in 4 Norwegian production facilities. dph: days post-hatch. $\div$ : not used

\begin{tabular}{|lcccc|}
\hline & \multicolumn{5}{c}{ Production facility } \\
\cline { 2 - 5 } & 1 & 2 & 3 & 4 \\
\hline Temperature range $\left({ }^{\circ} \mathrm{C}\right)$ & $8-10$ & $7-11$ & $7-10$ & $6-8$ \\
Rotifers (dph) & $1 \rightarrow 36$ & $1 \rightarrow 37$ & $1 \rightarrow 38$ & $1 \rightarrow 4$ \\
Artemia sp. (dph) & $\div$ & $22 \rightarrow 70$ & $20 \rightarrow 75$ & $\div$ \\
Natural zooplankton (dph) & $\div$ & $\div$ & $\div$ & $1-80$ \\
Formulated feed (dph) & $17 \rightarrow$ & $22 \rightarrow$ & $22 \rightarrow$ & $70 \rightarrow$ \\
Initial density of larvae $\mathrm{l}^{-1}$ & 120 & 100 & 90 & 100 \\
Survival (\%) & 4 & 8 & 10 & 1 \\
\hline
\end{tabular}


dehydrated in ethanol and embedded in methacrylate (Technovit 7100; Heraeus Kulzer). Sections, cut to a thickness of $1.5 \mu \mathrm{m}$, were mounted on glass slides and stained with toluidine blue (Philpott 1966). Digital micrographs were made using a ProgRes C14 camera (Jenoptik) on an Olympus Vanox AHBT3 microscope (Olympus Optical), and the images were processed using Adobe Photoshop.

Bone staining of whole specimens. Before staining, the specimens were fixed for at least $48 \mathrm{~h}$ in $4 \%$ formaldehyde in phosphate buffer ( $\mathrm{pH}$ 7.4). The fish were immersed in a buffered enzyme solution for up to $5 \mathrm{~d}$, depending on their size. This solution contained $0.4 \mathrm{mg} \mathrm{ml}^{-1}$ of trypsin (Sigma Chemical), Proteinase K (E. Merck), pronase (E. Merck) and lipase (Sigma Chemical). They were then washed in buffer and bleached in $0.3 \% \mathrm{H}_{2} \mathrm{O}_{2}$ in $1 \% \mathrm{KOH}$ for 1 to $4 \mathrm{~h}$, depending on their size. The bone matrix was then stained with $0.1 \mathrm{mg} \mathrm{ml}^{-1}$ Alizarin red S (CI 58005) in $1 \% \mathrm{KOH}$ for 24 to $48 \mathrm{~h}$, depending on the size of the sample, and then dehydrated and stored in glycerol. Digital micrographs were made using a Sony DXCS500 camera (Sony) on a Leica MZ16 A stereomicroscope (Leica Microsystems), and the images were processed using Adobe Photoshop.

\section{RESULTS}

\section{Normal development}

Individuals that developed normally had a straight post-cranial notochord. The cranial portion of the notochord had a ventral inclination, was funnelshaped, and the tip terminated directly caudal to the pituitary gland. Together with the parachordal cartilages, this portion of the notochord formed the floor of the neurocranium. In the vertebral column, the first structure to form was the bone of the foremost neural arch, which was observed 21 dph (Fig. 2). Thereafter, the neural arches were formed in bone, successively in a caudal direction. Mineralisation of the chordacentra first appeared in the cranial part of the prospective vertebral column $28 \mathrm{dph}$, and they mineralised in a regular sequence from here towards the tail fin (Figs. 3 to 5). All the vertebrae were formed $52 \mathrm{dph}$ (Fig. 6). Histological observations showed that the chordacentra were formed through mineralisation of the preformed notochordal sheath. The arches and the bone of the vertebral bodies were formed through direct ossification, and thus no cartilaginous tissue was present.

The initial ventral inclination of the floor of the neurocranium, including the cranial portion of the notochord, progressively diminished during development to the juvenile stage, finally becoming horizontal and aligned with the vertebral column. Thus, the fully developed axial skeleton has no curvatures and is horizontally aligned with the floor of the neurocranium.

\section{Malformation}

Fish displaying malformation showed a sequential and spatial pattern of skeletal development similar to that of normal specimens. No abnormalities were observed in the notochord during the yolk-sac period, which lasts approximately $1 \mathrm{wk}$. Deformation of the notochord was first observed during the transition to live feed, from approximately $7 \mathrm{dph}, 2$ wk before formation of the bony components of the vertebral column was initiated. During this period an abnormal dorsal curvature of the notochord in the region just behind the cranium, adjacent to the swim bladder, was observed in most specimens (Figs. 7 to 9). In this region the notochord also had an abnormal cross-sectional outline, characterised by a groove-shaped, longitudinal impression along the ventral surface of the sheath (Fig. 10).

Some floating fish were observed in all groups, and histological examination in some of these cases revealed an abnormally large swim bladder. In some groups, fish with a severely distended abdomen due to overfilling of the digestive tract with large quantities of feed particles were observed (Fig. 11). In one group, the intestine was full of fluid with a high content of bacteria, and gas bubbles were sometimes observed in the gut. Histological examination showed that the bacteria were curved rods (Vibrio-like).

In most cases, the swim bladder filled the impression (Fig. 10), and in severely affected larvae, it formed a hernia into the notochord (Fig. 12). A continuous range of dorsal curvatures and impressions, from mild to severe, was observed. These deformations persisted during the period in which the development of the vertebral column commenced.

The dorsal curvature of the notochord in the region above the swim bladder seemed to coincide with an abnormal formation of the neural arches, which were dorsoventrally compressed, adopting an S-shape (Figs. 13 \& 14). The angle between the neural arches and the vertebral bodies was thus affected, and the arches on the most cranial vertebrae frequently displayed an increased caudally oriented angle. The arches of the vertebrae in the caudal part of the malformed region often had an increased cranial inclination (Figs. 13 \& 14). In addition, the most severely affected neural arches did not fuse apically to form spinal processes. 


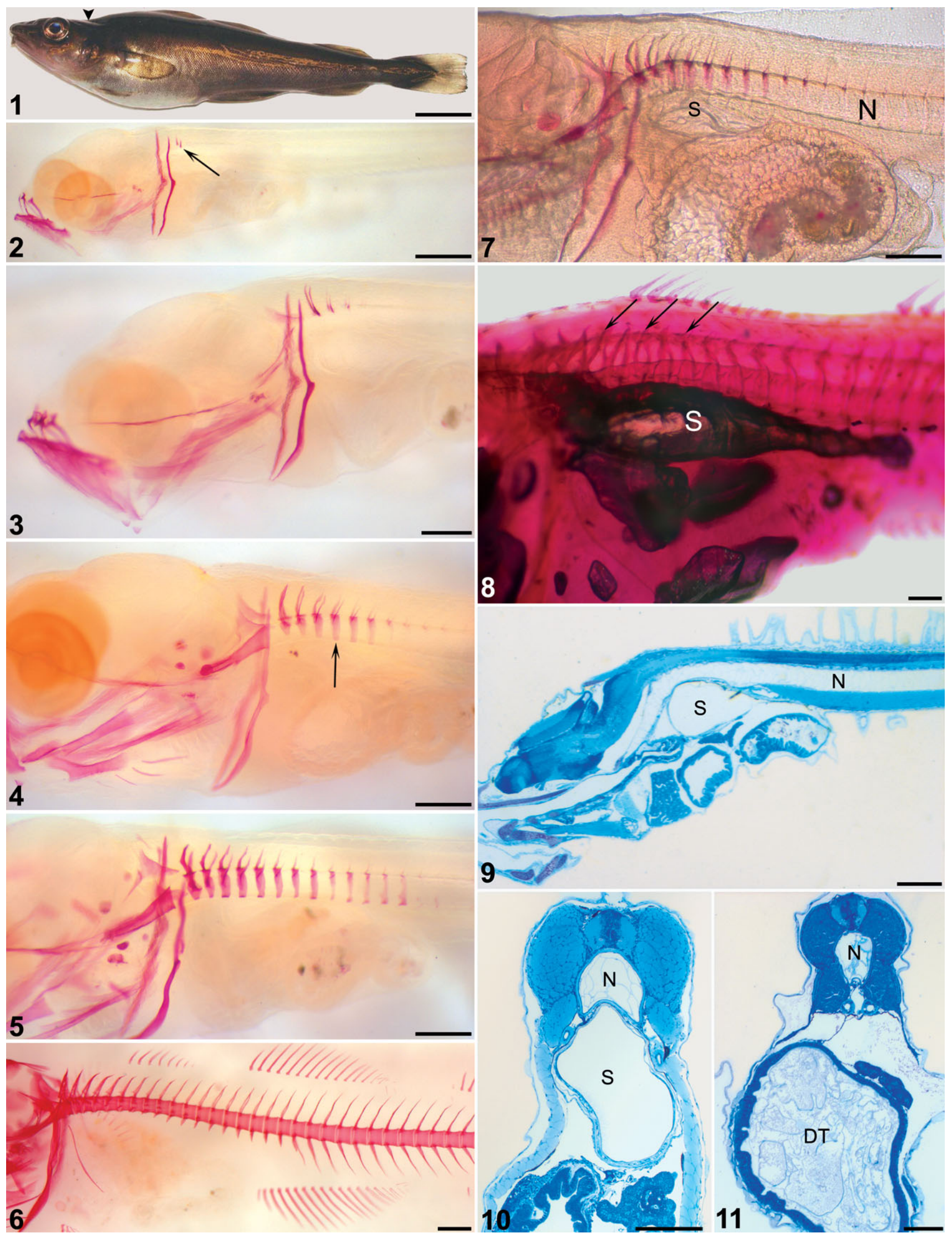


Figs. 1 to 11. Gadus morhua. Fig. 1. Juvenile cod $(50 \mathrm{~g})$ showing malformation characterised by slight upward tilt of head and indented dorsal contour at transition between head and trunk (arrowhead) (scale bar $=1 \mathrm{~cm}$ ). Figs. 2 to 8. Whole larvae stained with Alizarin red. Fig. 2. Normal larvae at $21 \mathrm{~d}$ post-hatch (dph); the most cranial neural arch anlagen has been formed (arrow) (scale bar $=500 \mu \mathrm{m}$ ). Fig. 3. Normal larvae at $24 \mathrm{dph}$; neural arch anlagen of the 3 most cranial vertebrae can be observed on the dorsal surface of the notochord (scale bar $=200 \mu \mathrm{m}$ ). Fig. 4 . Normal larva at $28 \mathrm{dph}$ showing formation of the first 7 vertebrae, in addition to neural arch anlagen, thin mineralised rings (chordacentra) have formed within the notochordal sheath (arrow); chordacentra are initially mineralised on the dorsal side of the sheath, after which the process of mineralisation proceeds bilaterally in a ventral direction $($ scale bar $=250 \mu \mathrm{m}$ ). Fig. 5 . Normal larva at $31 \mathrm{dph}$; anlagen of the 13 first vertebrae are visible $($ scale bar $=$ $300 \mu \mathrm{m}$ ). Fig. 6. Normal larva at $52 \mathrm{dph}$; all elements that comprise adult vertebral column have been formed; note that neural and haemal arches on adjacent vertebrae are almost parallel (scale bar $=300 \mu \mathrm{m}$ ). Fig. 7 . Larva at $44 \mathrm{dph}$ with malformation; image is illuminated to show soft tissues; note abnormal dorsal curvature in the notochord (N) just caudal to the cranium, adjacent to the swim bladder $(\mathrm{S})$; contour of the swim bladder is visible on ventral side of the notochord (scale bar $=300 \mu \mathrm{m}$ ). Fig. 8 . Larva at $59 \mathrm{dph}$, showing relationship between the swim bladder $(\mathrm{S})$ and the malformed region of the vertebral column; in the region above the swim bladder, neural arches are compressed and show abnormal inclinations (arrows) (scale bar $=150 \mu \mathrm{m}$ ). Figs. 9 to 11. Histological sections of larvae embedded in methacrylate and stained with toluidine blue. Fig. 9. Longitudinal section of larva at $20 \mathrm{dph}$; note abnormal curvature of the notochord (N) over the inflated swim bladder (S) (scale bar $=100 \mu \mathrm{m}$ ). Fig. 10. Transverse section through the middle region of the swim bladder of larva at $20 \mathrm{dph}$; note abnormal cross-sectional outline of the notochord $(\mathrm{N})$; the swim bladder (S) fills a longitudinal grove on the ventral side of the notochord (scale bar $=100 \mu \mathrm{m}$ ). Fig. 11 . Transverse section of larva at $15 \mathrm{dph}$; note the pathologically distended digestive tract (DT) filled with feed particles; this section is from the abdominal region caudal to the swim bladder; in this region the notochord $(\mathrm{N})$ was not affected by the swim bladder and had a normal cross-sectional outline (scale bar $=100 \mu \mathrm{m})$

The ventral, longitudinal impression of the notochord was associated with malformation of the chordacentra that attained the same abnormal cross-sectional profile as the notochord. In addition, the increased dorsal curvature of the notochord coincided with formation of ventrally wedge-shaped chordacentra. The 10 first vertebrae, especially the first 4, subsequently had an abnormal growth pattern (Figs. 13 \& 14). The vertebrae in the region behind the swim bladder had normal morphology.

Affected fish developed a marked dorsal curvature in the cranial part of the vertebral column (Figs. 15 to 17 ), and in severe cases a compensatory ventral curvature was observed in the transitional region between the abdominal cavity and the tail. In some specimens the neurocranium was malformed and radiographs showed that the neurocranium floor was curved, and the articulation between the vertebral column and the cranium was displaced to a more dorsal position (Figs. 16 \& 17). The most cranial segments of the epaxial lateral muscles seemed compressed and were not fully developed.

Malformation was observed in all the groups, with prevalence in the range of 70 to $90 \%$. A comparison between normal development and malformation, which summarises our main results, are presented in Figs. 18 to 21 .

\section{DISCUSSION}

The pathogenesis of the malformation of the neurocranium and the most cranial region of the spine seems to involve deformation of the notochord, leading to subsequent malformation of the chordacentra, verte- bral bodies and neural arches in the region above the swim bladder. The swim bladder appears in turn to play a role by exerting or transmitting an upward mechanical force that deforms the notochord. This pressure is likely to persist during the mineralisation of the chordacentra, if the notochordal deformation is to be conveyed to the vertebral column. Since the chordacentra act as foundations for the direct ossification that forms the vertebral bodies (Grotmol et al. 2003), malformed chordacentra will result in abnormal morphogenesis of the vertebrae. Moreover, during growth, the abnormal shape of the vertebral bodies may become more pronounced, thus increasing the dorsal curvature of the vertebral column. Typically, the malformed chordacentra were wedge-shaped and this was transmitted to the vertebral bodies, which thus did not form a straight row. The critical time window for development of malformation thus seems to be the period from 18 to $36 \mathrm{dph}$, during which the chordacentra and the neural arches are formed.

Because of the malformation of the vertebral bodies, the abnormal dorsal curvature first observed in the notochord may have persisted, and as a consequence the normal process of straightening of the axis between the floor of the neurocranium and the vertebral column may have been obstructed. A combined effect of the malformation of the neurocranium, the abnormal angle of articulation between the cranium and vertebral column, and the dorsal curvature of the vertebral column may have altered the spatial conditions required for normal development of the most cranial segments of the epaxial lateral muscles. This may have contributed to the indented dorsal body contour.

The pathological mechanisms that underlie the impact of the swim bladder on the notochord remain to be 


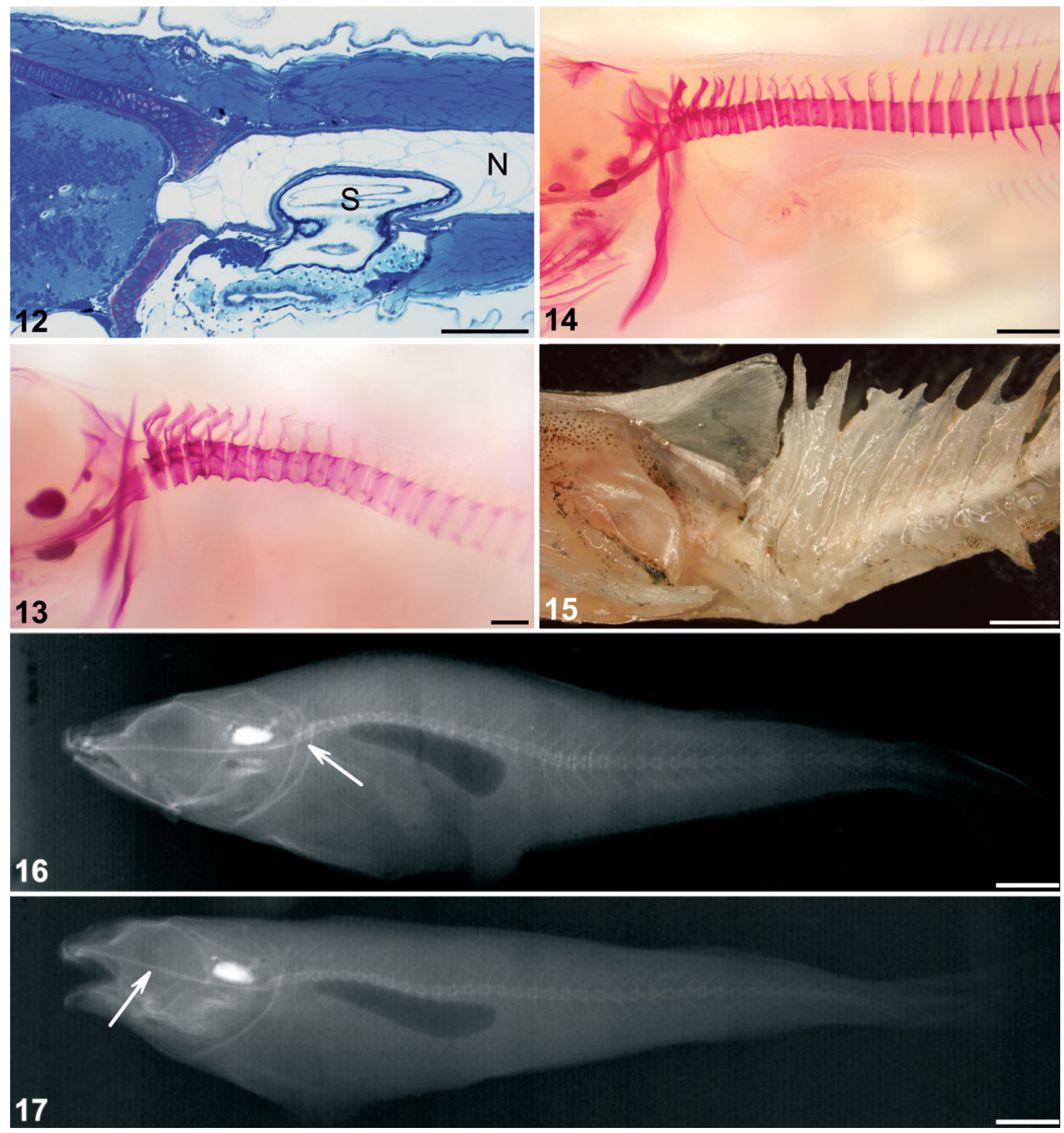

Figs. 12 to 17. Gadus morhua. Fig. 12. Histological longitudinal section of larva at 20 dph embedded in metacrylate and stained with toluidine blue, showing severe deformation of the notochord $(\mathrm{N})$ by the swim bladder (S), which forms a hernia into the notochordal tissue, nearly penetrating it (scale bar $=100 \mu \mathrm{m}$ ). Figs. 13 \& 14. Whole larvae stained with Alizarin red. Fig. 13. Larva at 56 $\mathrm{dph}$; severe malformations evident in the vertebral region dorsal to the swim bladder; note non-parallel neural arches and the wedge-shaped vertebral bodies with ventral concavity (scale bar $=200 \mu \mathrm{m}$ ). Fig. 14. Larva at $52 \mathrm{dph}$; note typical regional deformity in the 13 most cranial vertebrae; neural arches are non-parallel, with an abnormal shape, and vertebral bodies are wedgeshaped with a ventral concavity (scale bar $=300 \mu \mathrm{m})$. Fig. 15. Dissected juvenile $(50 \mathrm{~g})$ with malformation; most of the soft tissues have been removed, and the preparation shows the caudal part of the neurocranium and the cranial region of the vertebral column; normally at this stage of development the floor of the neurocranium and the vertebral column are aligned; note abnormal angle between the head and the vertebral column, and the dorsal curvature of the vertebral column (scale bar $=2 \mathrm{~cm})$. Figs. 16 \& 17. Lateral radiographs of juveniles $(15 \mathrm{~g})$ with malformation. Fig. 16. Caudal part of the floor of the neurocranium has a dorsal curvature that results in dorsal displacement of the occipital joint (arrow); this specimen also displays a marked curvature in the abdominal region of the vertebral column (scale bar $=1 \mathrm{~cm}$ ). Fig. 17. Neurocranium is severely malformed, and the floor has an abnormal dorsal inclination (arrow); in addition, the occipital joint is dorsally displaced and the vertebral column is sigmoid, with a dorsal curvature in the cranial region and a presumably compensatory ventral curvature more caudally $(\mathrm{scale}$ bar $=1 \mathrm{~cm}$ ) 

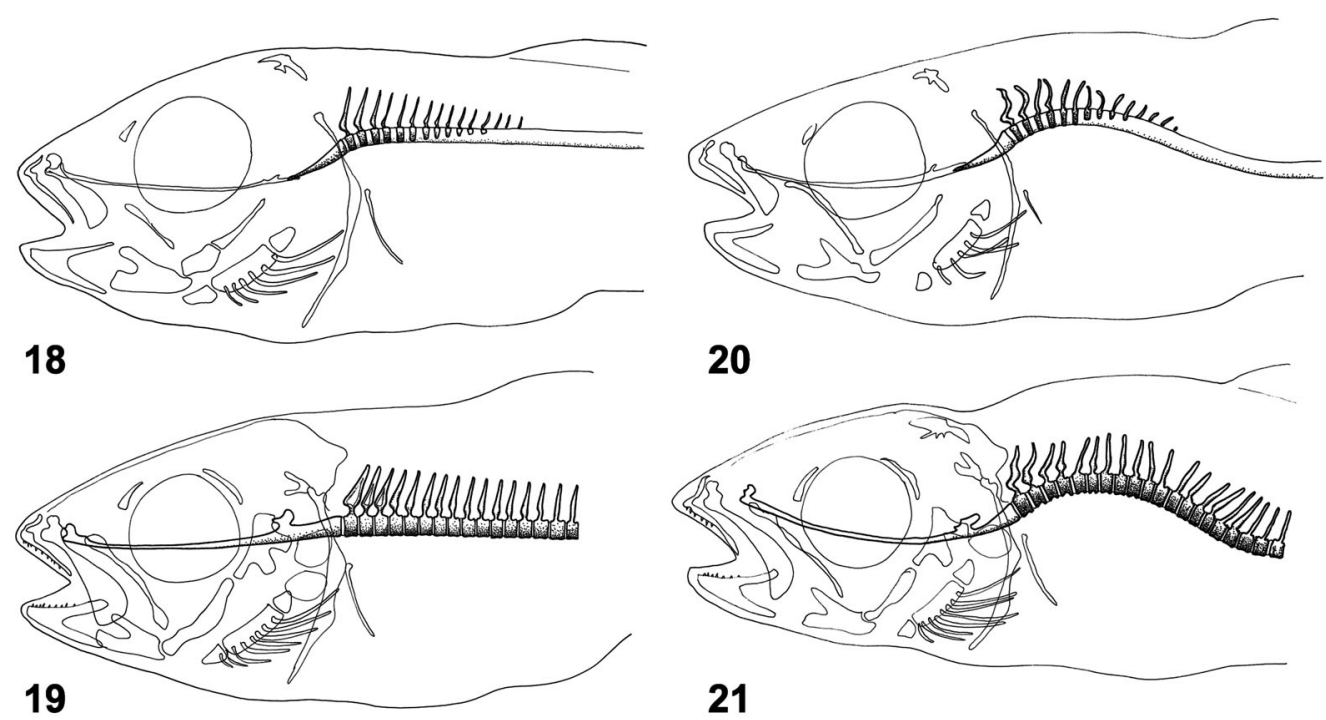

Figs. 18 to 21. Gadus morhua. Line drawings that schematically illustrate normal skeletal development in cod compared to malformation; the figures are not to scale. Fig. 18. Illustration of shape and course of the notochord with some vertebral anlagen in a normal larva at $28 \mathrm{dph}$; cranial portion of the notochord has a ventral inclination, while the post-cranial region is straight. Fig. 19. Initial ventral inclination of the floor of the neurocranium, including the cranial portion of the notochord, which progressively diminishes during development to the juvenile stage, finally becoming horizontal and aligned with the vertebral column; thus, the fully developed vertebral column has no curvatures and is horizontally aligned with the floor of the neurocranium. Fig. 20. Illustration of shape and course of the notochord with vertebral anlagen in a larva with deformation of the notochord (28 dph); note the postcranial dorsal curvature of the notochord. Fig. 21. Typical deformation of the floor of the neurocranium and the vertebral column

elucidated. Increased pressure between the notochord and the swim bladder may be caused by a chronic overinflation of the swim bladder itself or result from transmission of pressure through the swim bladder from pathologically expanded abdominal organs, such as an overfilled digestive tract. Apart from this, it cannot be excluded that reduced hydrostatic pressure (turgor) within the notochord may make it prone to deformation.

Our observations indicate that, in some cases, overinflation of the swim bladder may have produced the pressure on the notochord. Under intensive culture conditions, cod larvae and juveniles seem to be vulnerable to overinflation of the swim bladder (King \& Nardi 2002, Shields et al. 2003), and such overinflation has also been reported in other species (Johnson \& Katavic 1984, Bagarinao \& Kungvankij 1986, Noga 1996). The pathogenesis, however, has not been established. It has been claimed that cod larvae are hypersensitive to moderate levels of gas supersaturation $(<119 \%$ total gas pressure), which may cause overinflation of the swim bladder (King \& Nardi 2002).

A sudden decrease in ambient pressure could also induce overinflation. During the critical time window, aeration from the bottom of the tank is often employed to produce rapid upwelling currents in order to impede sedimentation of live feed. Larvae on the bottom may be caught in these upwellings, and be drawn rapidly to the surface. Such a vertical movement of $1 \mathrm{~m}$ or more will result in a decrease in ambient pressure of about $10 \%$ and a corresponding increase in the swim bladder volume.

The initial inflation of the swim bladder in cod larvae usually occurs by 5 dph (King \& Nardi 2002). Although there are numerous studies on the structure and function of the adult swim bladder in cod, little is known about this organ in the larvae. Our observations indicate that, as in haddock (Schwartz 1971), a functional gas-gland-like epithelium with a rete mirabile and an open pneumatic duct is present at the time of initial inflation. Whether the initial inflation occurs by intake of air from the surface through a pneumatic duct or by glandular production of gas has not been determined (Hardy 1978, Doroshev et al. 1981, Morrison 1993). Furthermore, it is not known when the gas gland and the oval (area for gas resorption) first become functional, and how pressure is regulated in the larvae. Because of this lack of knowledge, a rationale capable of relating culturing conditions to swim bladder overinflation is difficult to construct, and further studies are thus needed to elucidate the pathophysiology of the swim bladder of cod larvae.

The overfilling of the digestive tract and the extreme distension of the abdomen observed in several groups in this study during the critical time window for malfor- 
mation may have caused increased pressure between the swim bladder and the notochord. The overfilling of the digestive tract with feed particles may have resulted from overfeeding or constipation, related to suboptimal feed delivery protocols and/or diet. Large feed organisms like Artemia sp. nauplii may also have contributed to the overfilling. An abnormal intestinal flora with abundant growth of Vibrio-like bacteria, encountered in one of the groups, may have produced an abnormal influx of fluid into the intestinal lumen.

In conclusion, the characteristics of the malformation, such as deformed neural arches, wedge-shaped vertebral bodies with ventral concavity, and the abnormal alignment of the axis between the neurocranium and the vertebral column, are compatible with the notion that the notochord and the anlagen of the vertebral column have been forced upwards in the region above the swim bladder. A causal relationship, however, between the observed malformation and the filling of the swim bladder and/or the digestive tract has yet to be established. The pathomorphology described herein may be employed as a descriptor to outline cause-and-effect relationships between this type of malformation and the conditions of cultivation.

Acknowledgements. We thank Teresa Cieplinska and Nina Ellingsen for excellent technical assistance. The Research Council of Norway and Innovation Norway AS supported this project.

\section{LITERATURE CITED}

Arratia G, Schultze HP, Casciotta J (2001) Vertebral column and associated elements in dipnoans and comparison with other fishes: development of homology. J Morphol 250: 101-172

Bagarinao T, Kungvankij P (1986) An incidence of swim bladder stress syndrome in hatchery-reared sea bass (Lates calcarifer) larvae. Aquaculture 51:181-188

Baskerville-Bridges B, Kling LJ (2000a) Larval culture of Atlantic cod (Gadus morhua) at high stocking densities. Aquaculture 181:61-69

Baskerville-Bridges B, Kling LJ (2000b) Early weaning of Atlantic cod (Gadus morhua) larvae into a microparticulate diet. Aquaculture 189:109-117

Doroshev SI, Cornacchia JW, Hogan K (1981) Initial swim bladder inflation in the larvae of physoclistous fishes and

Editorial responsibility: Thomas Lang,

Cuxhaven, Germany its importance for larval culture. In: Lasker RM, Sherman $\mathrm{K}$ (eds) The early life history of fish: recent studies. J Cons Int Explor Mer 178:495-500

François Y (1966) Structure et développement de la vertèbre de Salmo et des téléostéens. Arch Zool Exp Gén 107:287-328

Goodrich ES (1930) Studies on the structure and development of vertebrates. Macmillan, London

Grotmol S, Kryvi H, Nordvik K, Totland GK (2003) Notochord segmentation may lay the pathway for the development of the vertebral bodies of the Atlantic salmon Salmo salar. Anat Embryol 207:263-272

Hardy JD (1978) Development of fishes of the mid-Atlantic Bight. An atlas of egg, larval and juvenile stages. II. Anguillidae through Syngnathidae. Fish Bull Fish Wildl Serv US 78/12:236-259

Howell BR (1984) The intensive culture of juvenile cod, Gadus morhua L. Flødevigen Rapp 1:657-675

Johnson DW, Katavic I (1984) Mortality, growth, and swim bladder stress syndrome of sea bass (Dicentrarchus labrax) larvae under varied environmental conditions. Aquaculture 38:67-78

King NJ, Nardi C (2002) Hatchery production of Atlantic cod: addressing nitrogen, oxygen and total gas pressure during larviculture. Bull Aquac Assoc Can 102:12-17

Koehl MAR, Quillin KJ, Pell CA (2000) Mechanical design of fiber-wound hydraulic skeletons: the stiffening and straightening of embryonic notochords. Am Zool 40:28-41

Laurence GC (1978) Comparative growth, respiration and delayed feeding abilities of larval cod (Gadus morhua) and haddock (Melanogrammus aeglefinus) as influenced by temperature during laboratory studies. Mar Biol 50:1-7

Morrison CM (1993) Histology of the Atlantic cod, Gadus morhua: an atlas. Part 4: Eleutheroembryo and larva. Can Spec Publ Fish Aquat Sci 119:496

Noga E (1996) Fish disease: diagnosis and treatment. MosbyYear Book, St. Louis, MO

Nordvik K, Kryvi H, Totland GK, Grotmol S (2005) The salmon vertebral body develops through mineralisation of two preformed tissues that are encompassed by two layers of bone. J Anat 206:103-114

Philpott DE (1966) A rapid method for staining plasticembedded tissues for light microscope. Sci Instr 11:11-12

Rosenlund G, Meslo I, Rodsjø R, Torp H (1993) Large-scale production of cod. In: Reinertsen H, Dahle L, Jørgensen L, Tvinnereim K (eds) Proceedings of the First International Conference of Fish Farming Technology, Norway, 9-12 Aug 1993. Balkema, Rotterdam, p 141-146

Schwartz A (1971) Swim bladder development and function in the haddock, Melanogrammus aeglefinus L. Biol Bull (Woods Hole) 141:176-188

Shields RJ, Irwin S, Smith PL, McEvoy LA (2003) Effects of diet transition regimen on survival, growth and lipid composition of intensively reared Atlantic cod, Gadus morhua, larvae. Aquacult Int 11:119-130

Submitted: June 4, 2004; Accepted: January 19, 2005

Proofs received from author(s): June 13, 2005 